\title{
Chua's Circuit for Experimenters Using Readily Available Parts from a Hobby Electronics Store
}

\section{Mr. Valentin Siderskiy, NYU Polytechnic School of Engineering}

Valentin Siderskiy received his B.Sc. degree from the NYU Polytechnic School of Engineering and M.Sc. degree from Columbia University. He conducts research in the Mechatronics and Controls Laboratory, where his interests include controls and chaos. Siderskiy is also the CTO of a technology startup.

\section{Mr. Aatif Ahmed Mohammed, NYU Polytechnic School of Engineering}

Aatif Mohammed is currently enrolled in his second year of B.S. Mechanical Engineering at NYU Polytechnic School of Engineering. He was a summer researcher at the Mechatronics and Controls Laboratory in 2014. Aatif was involved in the development of a cost effective version of the classic Chua's Circuit.

\section{Dr. Vikram Kapila, NYU Polytechnic School of Engineering}

Vikram Kapila is a Professor of Mechanical Engineering at NYU Polytechnic School of Engineering (SoE), where he directs a Mechatronics and Control Laboratory, a Research Experience for Teachers Site in Mechatronics and Entrepreneurship, a GK-12 Fellows project, and a DR K-12 research project, all funded by NSF. He has held visiting positions with the Air Force Research Laboratories in Dayton, OH. His research interests include K-12 STEM education, mechatronics, robotics, and control system technology. Under Research Experience for Teachers Site and GK-12 Fellows programs, funded by NSF, and the Central Brooklyn STEM Initiative (CBSI), funded by six philanthropic foundations, he has conducted significant K-12 education, training, mentoring, and outreach activities to integrate engineering concepts in science classrooms and labs of dozens of New York City public schools. He received NYUSoE's 2002, 2008, 2011, and 2014 Jacobs Excellence in Education Award, 2002 Jacobs Innovation Grant, 2003 Distinguished Teacher Award, and 2012 Inaugural Distinguished Award for Excellence in the category Inspiration through Leadership. Moreover, he is a recipient of 2014-2015 University Distinguished Teaching Award at NYU. In 2004, he was selected for a three-year term as a Senior Faculty Fellow of NYU-SoE's Othmer Institute for Interdisciplinary Studies. His scholarly activities have included 3 edited books, 7 chapters in edited books, 1 book review, 55 journal articles, and 109 conference papers. He has mentored 1 B.S., 16 M.S., and 4 Ph.D. thesis students; 31 undergraduate research students and 11 undergraduate senior design project teams; over $300 \mathrm{~K}-12$ teachers and 100 high school student researchers; and 18 undergraduate GK-12 Fellows and 59 graduate GK-12 Fellows. Moreover, he directs K-12 education, training, mentoring, and outreach programs that currently enrich the STEM education of over 1,100 students annually. 


\title{
Chua's Circuit for Experimenters Using Readily Available Parts from Hobby Electronics Stores
}

\begin{abstract}
In this paper we provide details on constructing the Chua's circuit out of readily available parts from two hobby electronics stores, specifically RadioShack and Fry's Electronics. The Chua's circuit is considered "simple" to construct provided necessary parts are available, however some items may be difficult to acquire. We provide instructions on how to compensate for difficult to find parts and tools. We also discuss the value of having a hands-on experiment for the study of the nonlinear systems, along with the importance of access to the tools and instructions for such an experiment.
\end{abstract}

\section{Introduction}

The Chua's circuit is considered to be one of the simplest to construct chaotic circuits. One application of constructing a Chua's circuit is to provide a hands-on experiment to aid in the study of nonlinear systems. Specifically, to provide a demonstration of bifurcation and hysteresis, one can tune various potentiometers in the circuit. Courses in which students may benefit from a live demonstration of a chaotic circuit include, but are not limited to, circuit theory, electronics, nonlinear dynamics and chaos, nonlinear differential equations, applied nonlinear control theory, dynamic system modeling, physiology, and others in departments such as electrical engineering, mechanical engineering, physics, biology, and mathematics. Whatever the application may be for constructing a Chua's circuit, it is possible to show the access to tools and instructions for common hobbyists, curious students, researchers, and educators.

Leon Chua is credited with developing the Chua's circuit in $1983^{1}$ and the circuit received considerable attention from the research community as a tool with simple structure to study chaotic systems. Several books and articles have been written on constructing Chua's circuits in several variations. ${ }^{2,3}$ The circuit is "simple" to construct, provided that all the necessary components are readily available along with all the measuring tools required to observe the chaotic attractor. The traditional Chua's circuit consists of three energy storing electrical components, specifically two 
capacitors and one inductor. Numerous papers have considered alternative designs of Chua's circuit to eliminate the inductor. ${ }^{4,5,6}$ Some reasons for this include:

- The changing state to be measured for an inductor is the current passing through it, which is difficult to measure accurately.

- The inductance value required to build the circuit may not be readily available.

- Often, the parasitic resistance of the inductor can be large enough to prevent the Chua's circuit to not function as intended.

A common technique is to replace the inductor with an inductor-gyrator circuit. ${ }^{5}$ Although Chua's circuit can be built readily by using available components, the observations of the attractor and nonlinear phenomena such as bifurcation are commonly done using an oscilloscope. However, hobbyists and students may not be able to afford their own oscilloscope since it can cost a few hundred to a couple of thousand dollars. An alternative inexpensive solution is to use the "LineIn" of a USB soundcard, which can provide two analog input signals with a sampling rate of 44 $\mathrm{kHz}$ or more.

In this paper, we present a Chua's circuit that can be constructed entirely out of components from a hobby electronics store (such as RadioShack and Fry's Electronics), and show that an external USB soundcard can be used as an inexpensive replacement to an oscilloscope and desktop soundcard. Several online resources are provided to diversify the channels through which one may best grasp the information. Such an approach will make chaotic systems more accessible and allow for their easy integration in student projects or classroom demonstrations. Finally, we discuss how this body of work is informed by a pedagogical perspective, and the value it has to individuals with varied learning styles.

\section{Chua's Circuit}

The Chua's circuit as shown in Figure 1 was originally designed not only to be simple to build but also simple in its dynamics. The design was intended to fulfill the minimum requirement of achieving chaos for a continuous time dynamical system. ${ }^{2}$ Various parameters of a Chua's oscillator include $L$ as a linear inductor, $R$ and $R_{0}$ as linear resistors, $C_{1}$ and $C_{2}$ as linear capacitors, and others that correspond to the Chua's diode. The state equations of the Chua's oscillator are given 


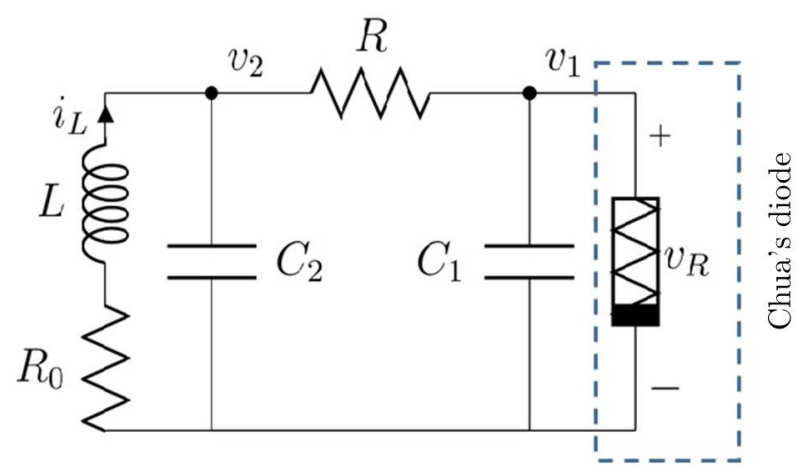

Figure 1: Chua's circuit schematic.

by

$$
\begin{aligned}
\frac{\mathrm{d} v_{1}}{\mathrm{~d} t} & =\frac{1}{C_{1}}\left(G\left(v_{2}-v_{1}\right)-g\left(v_{1}\right)\right), \\
\frac{\mathrm{d} v_{2}}{\mathrm{~d} t} & =\frac{1}{C_{2}}\left(G\left(v_{1}-v_{2}\right)+i_{L}\right), \\
\frac{\mathrm{d} i_{L}}{\mathrm{~d} t} & =\frac{1}{L}\left(-v_{2}-R_{0} i_{L}\right),
\end{aligned}
$$

where $G=1 / R$ and the nonlinearity $g\left(v_{R}\right)$ corresponding to the Chua's diode is characterized as follows

$$
g\left(v_{R}\right)= \begin{cases}G_{b} v_{R}+\left(G_{b}-G_{a}\right) E_{1}, & \text { if } v_{R} \leq-E_{1} \\ G_{a} v_{R}, & \text { if }\left|v_{R}\right|<E_{1} \\ G_{b} v_{R}+\left(G_{a}-G_{b}\right) E_{1}, & \text { if } v_{R} \geq E_{1}\end{cases}
$$

Although the schematic of Figure 1 is drawn in a fashion such that it is easy to comprehend how (1) and (2) arise from the shown interconnections, it is not obvious how to physically realize the circuit since some components may not be readily available off-the-shelf, e.g., the nonlinear component of the circuit, the Chua's diode. Moreover, as previously indicated, the implementation of an inductorgyrator is preferred in lieu of an inductor. We implement the Chua's diode schematic presented by Ref. 7 and the inductor-gyrator presented by Ref. 5 as shown in Figure 2. In most literature, the instructions for building a Chua's circuit do not go beyond the schematic and the parameters for each component. ${ }^{3}$ In the following section, we provide instructions for assembling Chua's circuits beyond the bare minimum of a schematic, including a resource to obtain the materials and instructions on assembling the circuit. 


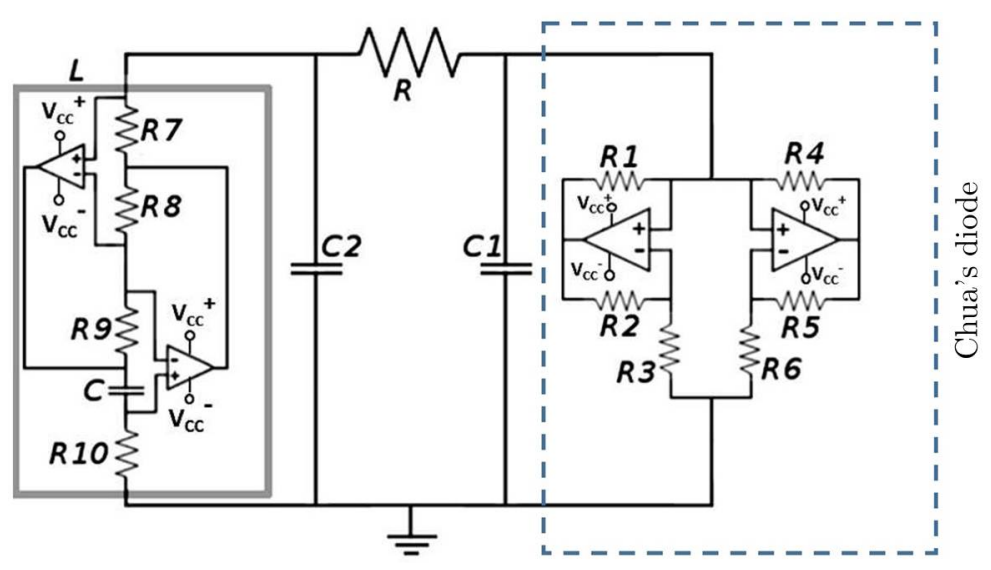

Figure 2: Chua's circuit schematic with realizable components.

\section{Building the Chua's Circuit from Hobby Electronics Store Parts}

Over the past three decades many hobby electronics stores, where one may have acquired all necessary components to build a Chua's circuit (except the inductor), have gone out of business in the United States. Franchises such as RadioShack and Fry's Electronics are the only locations where one may acquire electronic components (other than online ordering) but their selection of hobby electronic components is rather limited. Specifically, their stock for hobby electronics is currently geared to support programmable development platforms (e.g., Arduino and Raspberry Pi) instead of analog electronics.

With these limitations in mind, one major alteration to the schematic in Figure 2 had to be made as shown in Figure 3. Specifically, parameter values for $R$ and $R_{10}$ are ideally $2.5 \mathrm{k} \Omega$ potentiometers, however only $5 \mathrm{k} \Omega$ and $10 \mathrm{k} \Omega$ potentiometers are available that have large enough knobs to provide enough dexterity for the user to properly tune the circuit to observe bifurcation. As a solution, resistors $R_{11}$ and $R_{12}$ are placed in parallel to potentiometers $R_{10}$ and $R$, respectively, such that a similar range is achieved as a $2.5 \mathrm{k} \Omega$ potentiometer. In this new configuration, since the relation to the angle of the potentiometer knob and the equivalent resistance is not linear the best value for $R_{11}$ and $R_{12}$ was narrowed down via trial and error to easily produce bifurcation. In addition, the schematic in Figure 3 also shows how two batteries supply the power to the circuit with a power switch and power indicator LEDs.

Despite having a physically realizable schematic, it is the experience of the authors that this information alone is not sufficient to build a Chua's circuit without experiencing difficulties. There are several details that are frequently omitted in most literature. To remedy this situation, a web site has been created ${ }^{8}$ to promote and support building of Chua's circuits by hobbyist and students. The website is regularly updated based on suggestions from users and emails to the site administrator. 


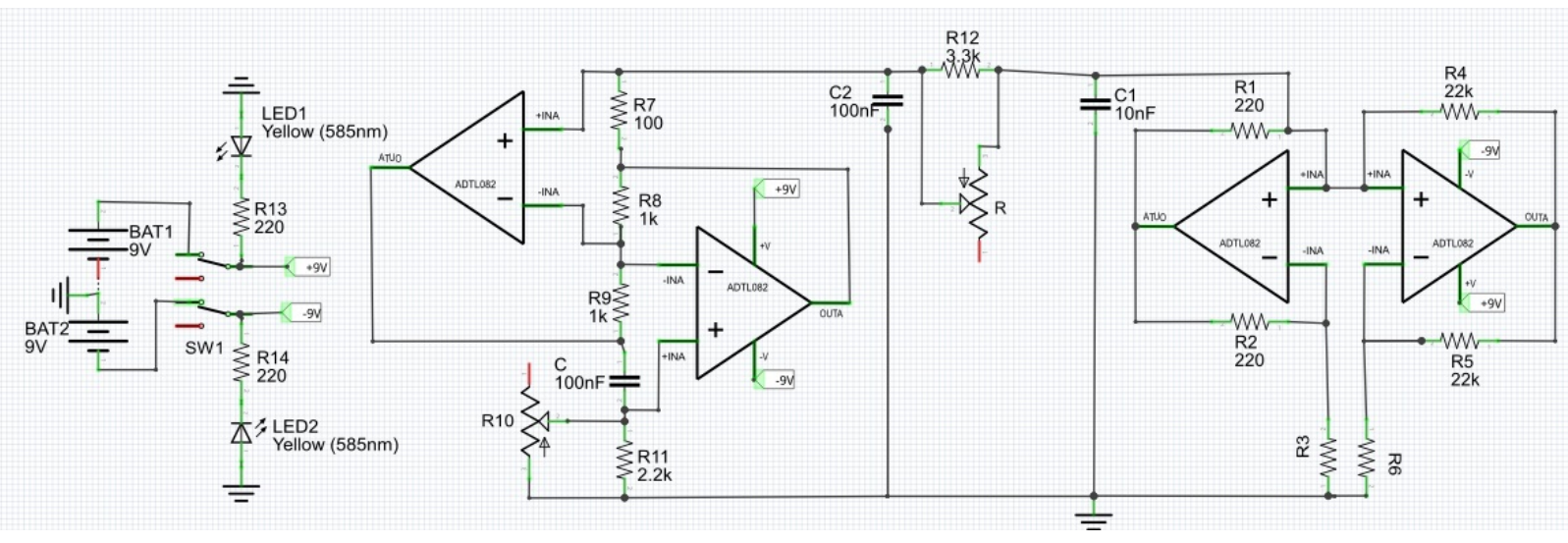

Figure 3: Chua's circuit schematic using Fritzing.

The list of potential pitfalls in creating a working circuit is long, especially for those who do not have a background in electrical circuits. An example includes the type of capacitors and batteries to use.

Tables 1 and 2 list the components required to build the Chua's circuit, selected from RadioShack and Fry's Electronics, respectively. The components that are available from RadioShack have the indicator "R" in the Source column, components that are available from Fry's Electronics have the indicator "F", and those that are available from other sources have the indicator "O". Tables 3 and 4 list the tools necessary to build the Chua's circuit, selected out of the inventories of RadioShack and Fry's Electronics, respectively. In many cases individual RadioShack or Fry's Electronics stores may not have all the necessary parts, hence for some parts alternative SKU numbers are also provided.

Due to the Maker movement, many resources have recently become available to build electronic circuits. This has raised the bar for quality and clarity of the presentation of projects, especially with the growth of Instructables a "website specializing in user-created and uploaded do-it-yourself projects, which other users can comment on and rate for quality". 9 The layout in which one makes an "Instuctable" on Instructables website is purposely restricted to posting a project in chronological steps. Each step starts with posted pictures and videos of that step and text is added at the bottom (often in very simple language and/or bullet points). Another resource that has grown in popularity is an open-source software project called Fritzing. ${ }^{10}$ One of its major features is a relatively easy interface to document breadboard layouts. Although this feature is not new, the company SparkFun has adopted Fritzing in many of its tutorials. The importance of these two tools is that they are both geared for visual learners and remove a layer of abstraction, specifically reading schematics or instructions in paragraph form. We have posted the instructions on how to make a Chua's circuit out of RadioShack components on Instructables ${ }^{11}$ and have adopted the use 
Table 1: RadioShack Chua's circuit component list.

\begin{tabular}{|l|r|r|r|r|r|}
\hline Description & Source & \multicolumn{1}{|c|}{ SKU } & Price & Qty & Price \\
\hline 9V Battery, Duracell Two Pack & $\mathrm{O}$ & N/A & 6.99 & 1 & 6.99 \\
9V Snap Connectors (5 PCK) & $\mathrm{R}$ & $270-324,270-325$ & 2.99 & 1 & 2.99 \\
Linear-Taper Potentiometer (10k or 5k) & $\mathrm{R}$ & $271-1715,271-1714$ & 3.49 & 2 & 6.98 \\
Resistors (500-Piece Value Pack) & $\mathrm{R}$ & $271-003$ & 10.99 & 1 & 10.99 \\
TL082 (8-PIN DIP) & $\mathrm{R}$ & TL082/TL082CP & 2.49 & 4 & 9.96 \\
0.01uF 10\% Film Capacitor & $\mathrm{R}$ & $272-1065,272-1051$ & 1.49 & 1 & 1.49 \\
0.1uF 10\% Film Capacitor & $\mathrm{R}$ & $272-1069,272-1053$ & 1.99 & 1 & 1.99 \\
DPDT Toggle Switch & $\mathrm{R}$ & $275-666$ & 3.99 & 1 & 3.99 \\
20 Assorted LEDs & $\mathrm{R}$ & $276-1622$ & 4.49 & 1 & 4.49 \\
Total & & & & & 49.87 \\
\hline
\end{tabular}

Table 2: Fry's Electronics Chua's circuit component list.

\begin{tabular}{|l|r|r|r|r|r|}
\hline Description & Source & Frys No. & Price & Qty & Price \\
\hline 9V Battery, Duracell Two Pack & F & 6467952 & 7.99 & 1 & 7.99 \\
9V Snap Connectors (5 PCK) & F & 136740 & 1.29 & 1 & 1.29 \\
Linear-Taper Potentiometer (10k or 5k) & F & 3065369,3067119 & 1.99 & 2 & 3.98 \\
Resistors (610 Assorted Value Pack) & F & 6349931 & 7.99 & 1 & 7.99 \\
NTE858M (8-PIN DIP) & F & 1004423 & 2.59 & 4 & 10.36 \\
0.01uF 10\% Film Capacitor & F & 999426 & 1.09 & 1 & 1.09 \\
0.1uF 10\% Film Capacitor & F & 999476 & 1.39 & 1 & 1.39 \\
DPDT Toggle Switch & F & 2860484 & 3.19 & 1 & 3.19 \\
10 Assorted LEDs & F & 452809 & 5.29 & 1 & 5.29 \\
Total & & & & & 42.57 \\
\hline
\end{tabular}

Table 3: RadioShack Chua's circuit tool list.

\begin{tabular}{|l|r|r|r|}
\hline Description & Source & \multicolumn{1}{|c|}{ SKU } & Price \\
\hline Hookup Wire (22AWG Solid) & $\mathrm{R}$ & $278-1221$ & 6.11 \\
Solderless Breadboard Jumper Wire Kit & $\mathrm{R}$ & $276-173$ & 5.94 \\
Breadboard & $\mathrm{R}$ & $276 \mathrm{WBU} 202$ & 16.49 \\
Wire Stripper/Cutter & $\mathrm{R}$ & 6400224 & 9.99 \\
Mini Long-nose Pliers & $\mathrm{R}$ & 6400062 & 7.49 \\
5-Piece Basic Soldering Set & $\mathrm{R}$ & $64-2802$ & 10.99 \\
Rosin Core Solder (2.5 Oz.) & $\mathrm{R}$ & $64-005$ & 5.09 \\
Total & & & 62.10 \\
\hline
\end{tabular}

of Fritzing to make schematics (Figure 3) and breadboard layouts (Figure 4) to accommodate visual learners. The use of aforementioned schematics result in a finished Chua's circuit as depicted in Figure 5. 
Table 4: Fry's Electronics Chua's circuit tool list.

\begin{tabular}{|l|r|r|r|}
\hline Description & Source & Frys No. & Price \\
\hline Hookup Wire (22AWG Solid) Set & F & 3018459 & 12.99 \\
Solderless Breadboard Jumper Wire Kit & F & 4612398 & 8.99 \\
Breadboard & F & 3849177 & 6.99 \\
Wire Stripper/Cutter & F & 6541403 & 9.99 \\
Mini Long-nose Pliers & F & 8026494 & 4.99 \\
Soldering Iron & F & 3317512 & 9.99 \\
60/40 No-Clean Solder, 0.032" Diameter, 0.5oz & F & 4455326 & 2.49 \\
Total & & & 56.43 \\
\hline
\end{tabular}

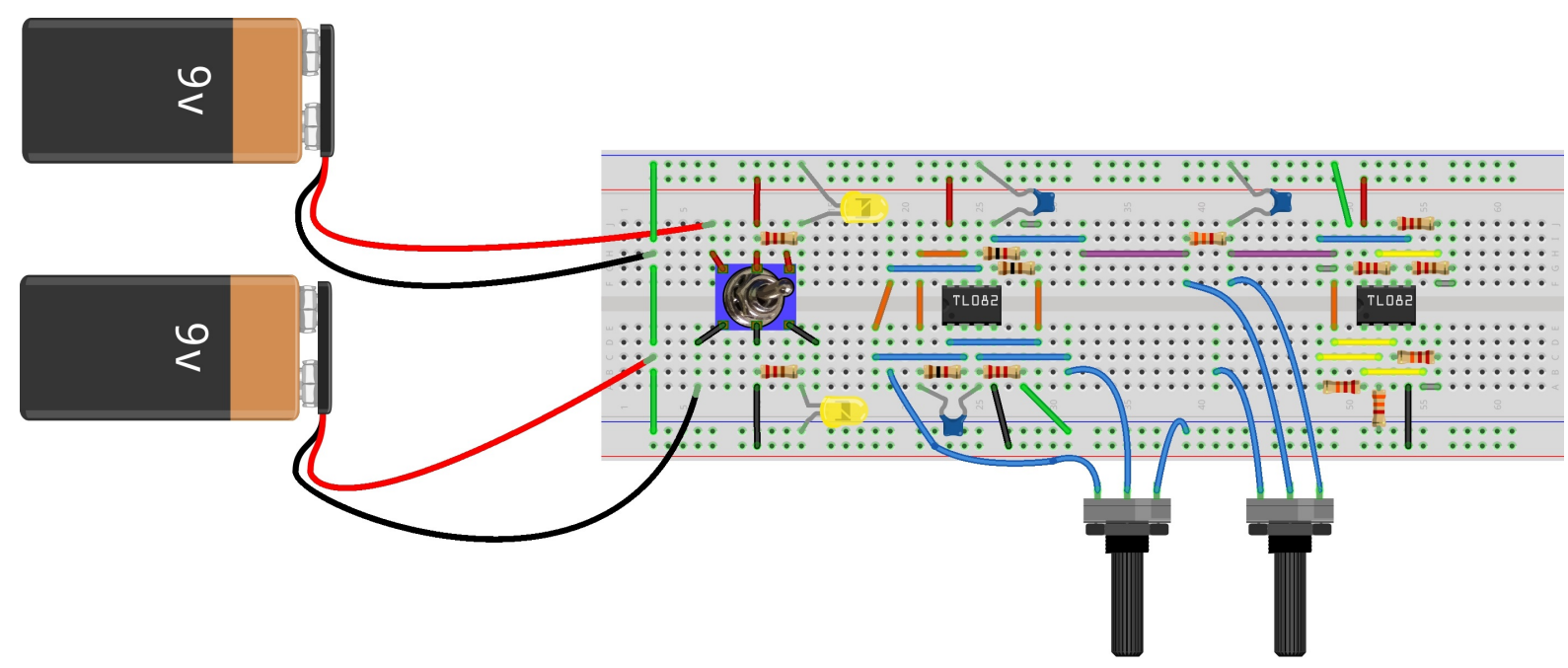

Figure 4: Chua's breadboard layout using Fritzing.

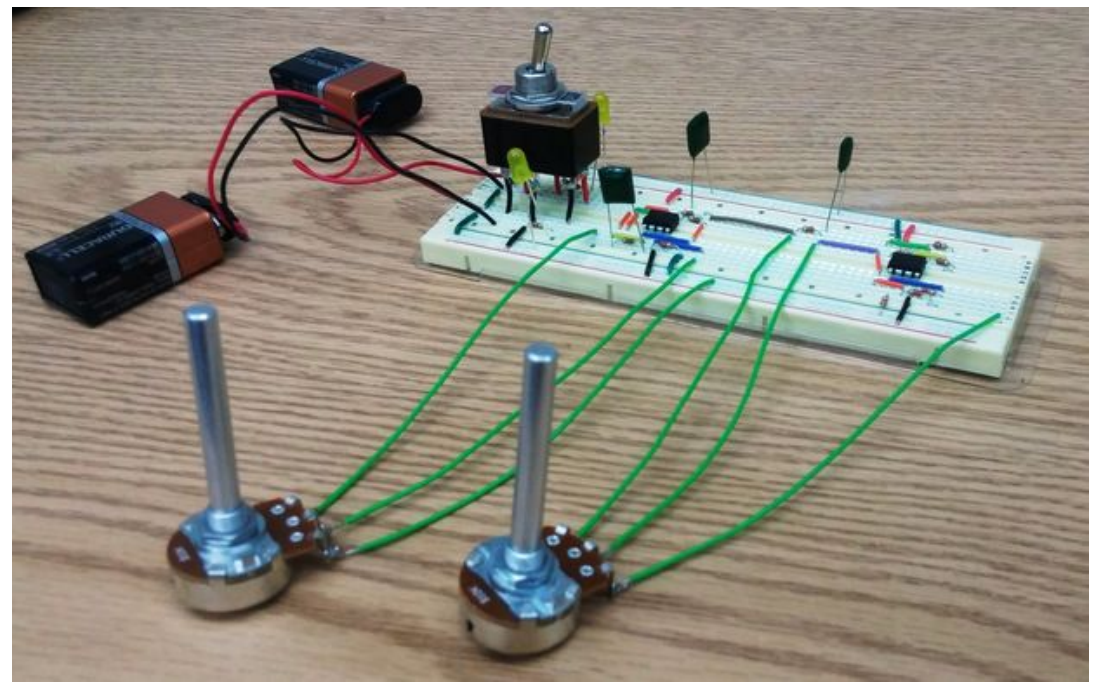

Figure 5: Fully constructed Chua's circuit. 


\section{USB Soundcard Scope}

In order to visualize the chaotic attractor one would typically use an oscilloscope (see Figure 6), however such devices are frequently out of the price range for hobbyists and students to make a simple demonstration. Moreover, the measurement of analog signals using a Line-In port of a desktop soundcard is no longer a relevant solution to replace an oscilloscope. The 1990's desktops came commonly equipped with a soundcard which included a Line-In port. The current computer market is split between desktop and laptop computers, and most laptops do not have a Line-In port. As an alternative, we propose using a USB soundcard such as the BEHRINGER U-CONTROL UCA222 that can achieve a sampling rate of $44 \mathrm{kHz}$ for two channels. Table 5 lists all the necessary components to make use of the USB soundcard to perform signal measurements on a Chua's circuit. Alternatively, Table 6 lists corresponding components available from Fry's Electronics. Note that a $1 \mathrm{M} \Omega$ resistor is to be connected between the Line-In and the Chua's circuit. Once the USB soundcard is connected, a user can utilize the two channels of Line-In as the XY-mode of an oscilloscope to produce phase plots. A recommended software for Windows users is Soundcard Oscilloscope developed by Christian Zeitnitz. ${ }^{12}$ Figure 7 is a screenshot of using Soundcard Oscilloscope while it is connected to the Chua's circuit and the chaotic attractor is clearly displayed. As one slowly tunes the potentiometer, the circuit response evolves from steady state and transitions into a chaotic mode as seen in Figure 8.

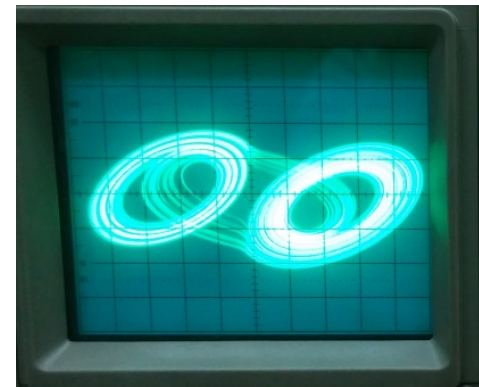

(a)

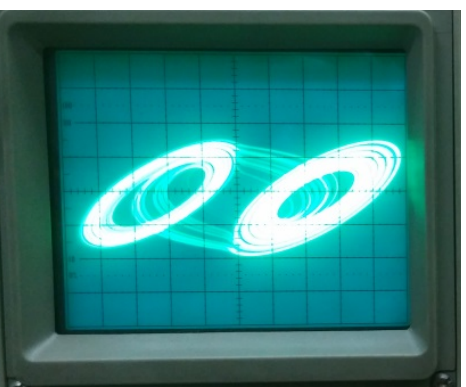

(b)

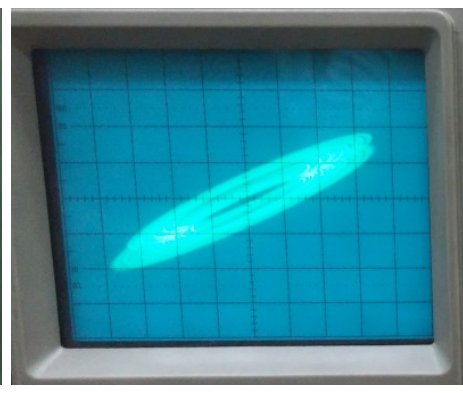

(c)

Figure 6: Chua's oscillator: (a) $v_{1}$ vs $v_{2}$, (b) $v_{1}$ vs $i_{L}$, and (c) $v_{2}$ vs $i_{L}$.

Table 5: RadioShack USB soundcard scope component list.

\begin{tabular}{|c|c|c|c|}
\hline Description & Source & SKU & Price \\
\hline 3 Ft. Stereo Patch Cable, Dual-RCA Plugs to Dual-RCA Plugs & $\mathrm{R}$ & RCA & 6.11 \\
\hline $14 "$ (35.3CM) Insulated Test/Jumper Leads & $\mathrm{R}$ & $278-1156$ & 8.99 \\
\hline BEHRINGER U-CONTROL UCA222 & $\mathrm{O}$ & UCA222 & 29.99 \\
\hline 1 Mega-ohm Resistor & $\mathrm{R}$ & $271-1356$ & 1.49 \\
\hline Total & & & 46.58 \\
\hline
\end{tabular}


Table 6: Fry's Electronics USB soundcard scope component list.

\begin{tabular}{|l|c|r|r|}
\hline Description & Source & Frys No. & Price \\
\hline Blackpoint 3' Dual RCA Cable & F & 1646487 & 2.79 \\
14" Colored Leads, Alligator Clips & F & 71912 & 9.49 \\
Diamond USB Xtreme Audio Adapter & F & 8020414 & 69.99 \\
1 Mega-ohm Resistor & F & 7307974 & 3.99 \\
& & Total & 86.26 \\
\hline
\end{tabular}

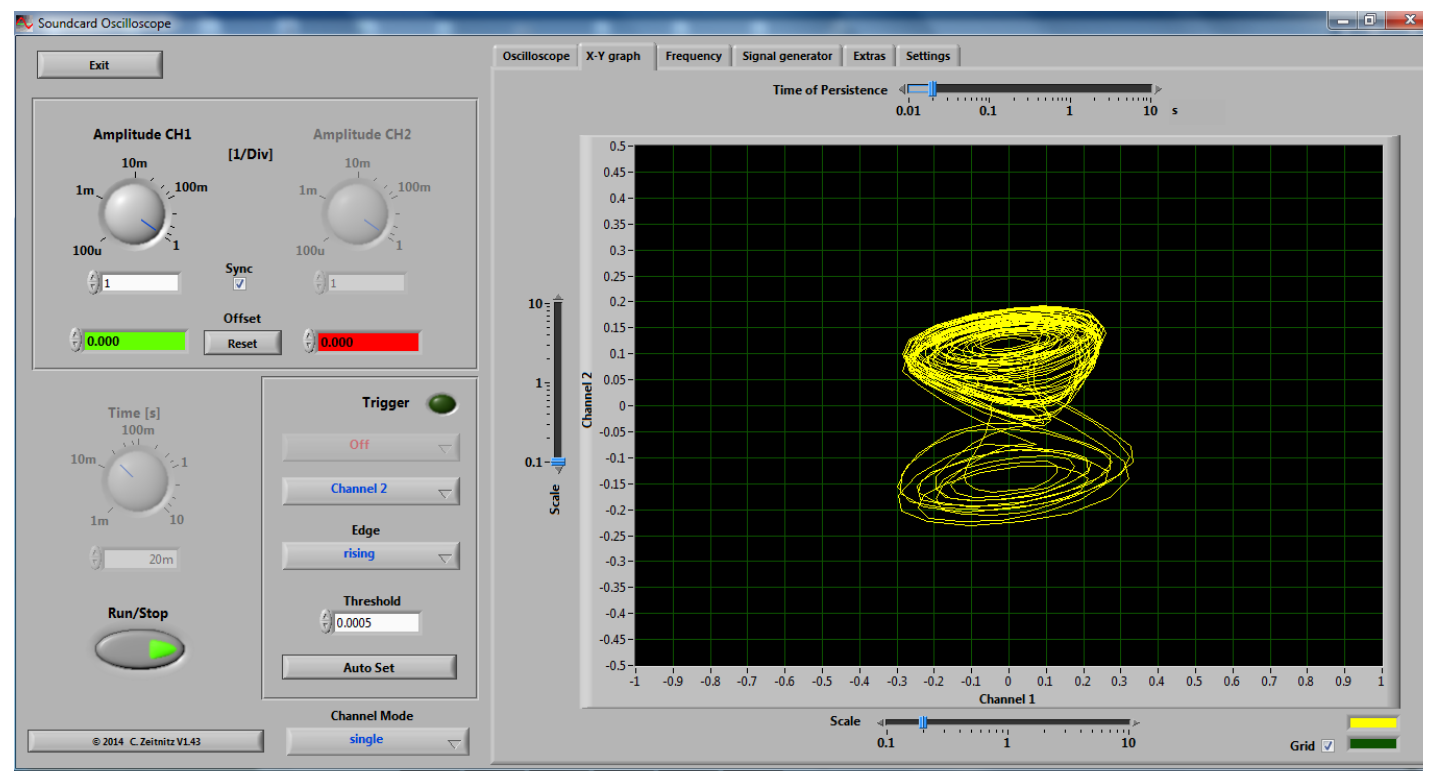

Figure 7: Software package, Soundcard Oscilloscope, is used to plot the phase portrait of the chaotic attractor.

\section{Learning Objectives and Pedagogy}

Laboratory experiments are widely used in engineering education to allow students to $i$ ) gain working familiarity with physical apparatus and manipulatives; $i$ i) gain experience with collecting, analyzing, and interpreting real data; and iii) gain an ability to connect fundamental engineering concepts with practical applications ${ }^{13}$ to develop a "feel for engineering". ${ }^{14}$ In this spirit, following learning objectives have been identified for this work. First, students will learn to interpret circuit diagrams, identify circuit components, and perform circuit assembly using a breadboard. Second, students will experimentally generate, read, and interpret a phase portrait. Third, students will produce and identify bifurcation behavior as a parameter is varied in the Chua's circuit. Fourth, students will identify hysteresis of the circuit output as a function of a changing parameter. Fifth, students will identify and interpret chaotic behavior by inspecting the chaotic circuit output.

Use of the Chua's circuit for instructional purposes in the classroom or laboratory is not uncommon. ${ }^{15,16,17}$ It can easily demonstrate nonlinear phenomena such as bifurcation and hysteresis as 


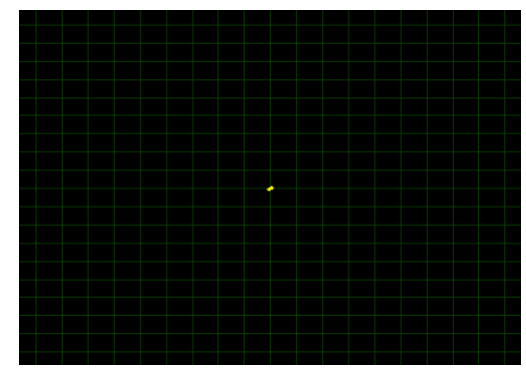

(a)

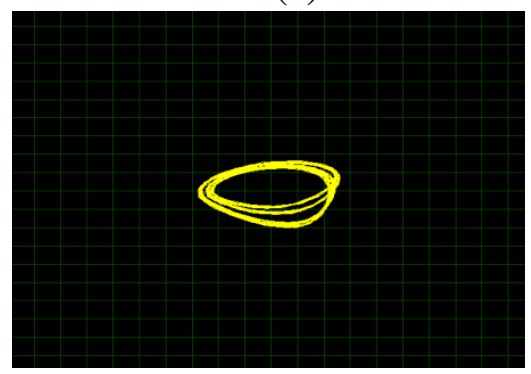

(d)

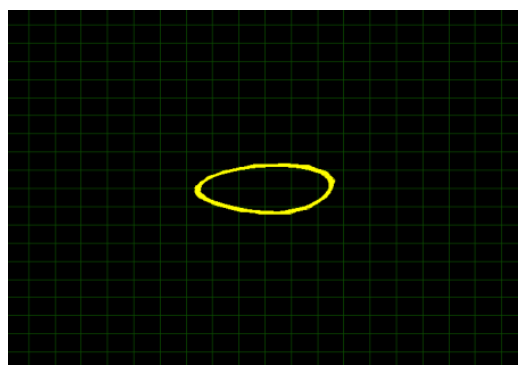

(b)

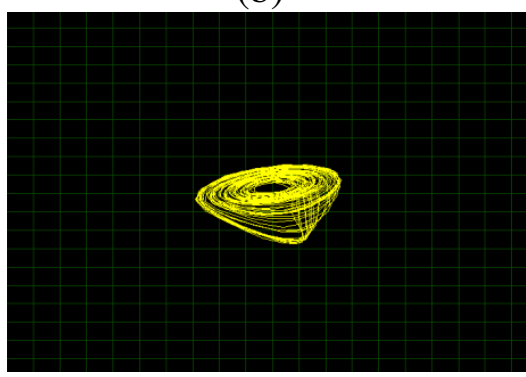

(e)

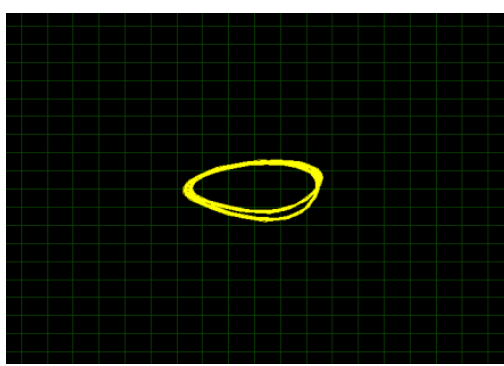

(c)

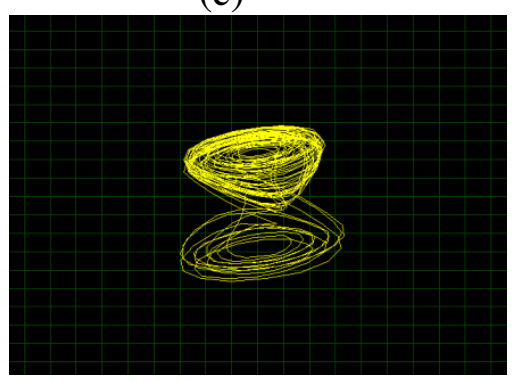

(f)

Figure 8: USB soundcard display: items (a)-(f) show how the display on the USB soundcard scope changes as a potentiometer is slowly turned on the Chua's circuit: (a) steady state; (b) period-1 limit cycle; (c) period-2 limit cycle; (d) period-4 limit cycle; (e) single scroll chaotic attractor; (f) double scroll chaotic attractor.

a parameter is varied. ${ }^{18}$ The students' experience is as follows: as students physically tune a potentiometer in the Chua's circuit, they will see the effect the turning has on the signal to bring a system from steady state, to a stable limit cycle, and to chaos. Then, when the knob is turned in the opposite direction, students will sense that there is not a one-to-one correspondence between the position of the knob of the potentiometer and the behavior of the circuit, witnessing hysteresis of the parameter.

Recent research has examined the effectiveness of physical experimentation versus simulation on learning outcomes. ${ }^{19,20}$ For example, even though situated and embodied characteristics of cognition intuitively suggest that physical experimentation and simulation will yield varied effects on student learning, ${ }^{21}$ in unconfounded experiments with elementary school students, physical and virtual materials have been shown to produce comparable effectiveness in accomplishing desired learning objectives. ${ }^{20}$ Alternatively, in a different study, participants engaged with physical experimentation achieved better learning gains than those who engaged with virtual experimentation. ${ }^{22}$ Although the nonlinear phenomena arising in the Chua's circuit can be demonstrated using computer simulations, physical experience can potentially be more valuable and vividly memorable to students of different learning styles, specially for kinesthetic learners with active touch and physical manipulation of experimental variables. There is value to students building their 
own experiments ${ }^{23}$ and hands-on laboratory experiments provide students with real data that includes dynamics of the whole system incorporating noise, parameter tolerances, parasitic effects, etc. ${ }^{24}$ Based on Felder and Silverman's model ${ }^{25}$ this experiment would be most fitting for sensory/visual/active learners. Moreover, in the context of Fleming's VARK model, ${ }^{26}$ this experiment is a good fit for the visual/kinesthetic learners.

For constructing the Chua's circuit, it may be of value to note that by providing instructions in various formats (schematics, breadboard layout, Instructables, tutorial website), students can possibly have diverse mechanisms to grasp the know-how of assembling a Chua's circuit in a manner that may be a natural fit for them. Yet, an exclusive reliance on visual tutorials (e.g., Instructables and Fritzing's breadboard layouts) may deprive a student from learning how to read a schematic. The decision as to which method is best to use for building a Chua's circuit can only be made based on the context. An electrical engineering graduate student studying nonlinear circuits would get more insight by reviewing the schematic diagram and differential equations of the system. In contrast, a demonstration of bifurcation as an analogy to a non-electrical engineering audience can benefit from being able to assemble the circuit quickly using a breadboard layout.

\section{Assessment}

Below we provide three examples in which high school, undergraduate, and graduate students were engaged in building, operating, observing, and analyzing the behavior of a Chua's circuit. The ability of students to successfully build the Chua's circuit and their feedback on obtained results provide a qualitative assessment that the aforementioned learning objectives were accomplished.

First, to illustrate that the framework of this paper can be readily used by undergraduate engineering students, the first and last authors of this paper recruited a mechanical engineering freshman, who had no prior experience with electronic circuits, as a summer intern. The student was tasked with building, testing, and demonstrating the Chua's circuit out of RadioShack components using the previously mentioned online resource Ref. 8. The student was able to accomplish the assigned task without any further direction and produced the steady state, limit cycle, chaos, and bifurcation behaviors, showing the validity of our proposed approach. The student, who is a co-author of this paper, provided feedback concerning the resources that were most helpful to build the Chua's circuit and recommended additional content and changes that have been compiled in the Instructables page. $^{11}$

Second, in a technical high school based in a suburban community, a presentation on chaos theory was given to 30 students enrolled in an introductory electrical and computer engineering class. 
The presentation used MATLAB simulations of a simple pendulum and a double pendulum to explain the properties of chaos such as sensitivity to initial conditions, dense periodic orbits, and the requirement that a system possess a certain degree of complexity to produce a chaotic orbit. The presentation followed a live demonstration of a chaotic attractor using the Chua's circuit connected to an oscilloscope. Students came up to the live demonstration to change the parameters of the Chua's circuit and to witness the bifurcation phenomena. After the presentation, the students in the class were provided 11 kits containing all the components needed to build a Chua's circuit and a link to online resource Ref. 8. To motivate the students, the teacher offered them extra credit for building a Chua's circuit on their own time by using various tools and measurement equipment available at the school. Since the visit to the class, four students have emailed the first author to indicate that each of them had constructed a functioning Chua's circuit. Moreover, they provided feedback with videos of their results and positive comments about the helpfulness of Ref. 8 to accomplish their task.

Third, in a graduate nonlinear dynamics class at a local university, to supplement a lecture that discussed hysteresis and nonlinear oscillators, two graduate students were provided Chua's circuits to create physical demonstrations to illustrate the concepts discussed in the classroom. One graduate student provided feedback that "playing with the circuit" provided him with a clearer understanding of hysteresis and "how it worked".

\section{Conclusion}

Access to physical tools and equipment is not growing as rapidly as our access to digital information. However, the information we share digitally offers novel ways to utilize our physical resources and the modes to share such resources are growing as well. These modes differ in the way content is shared or distributed and they appeal to different ways in which people consume and comprehend information. In this paper, we suggested a variety of methods to deliver a how-to-tutorial of constructing a Chua's circuit including a dedicated website and using tutorial platforms, Instructables and Fritzing. The tutorials take into account ease of access of components and include parts lists for walk-in hobby electronics stores (specifically, RadioShack and Fry's Electronics). The Chua's circuit itself can provide a valuable learning opportunity for the study of nonlinear systems as a hands-on experiment. Much of the current literature focuses on the treatment of nonlinear systems through lectures and computer simulations. This paper shows that the Chua's circuit can be readily adapted for hands-on active learning. 


\section{Acknowledgements}

This paper is supported in part by the following grants: NSF-DRL-1417769, NSF-EEC-1132482, NSF-DGE: 0741714, and NY Space Grant: 48240-7887. The authors would like to thank Richard Kroon, the editor and webmaster of www.chuacircuts.com, for providing assistance in developing the Chaos Circuit Instructables page.

\section{References}

[1] T. Matsumoto. A chaotic attractor from Chua's circuit. IEEE Trans. Circuits Sys., 31(12):1055-1058, 1984.

[2] L. Fortuna, M. Frasca, and M. Xibilia. Chua's Circuit Implementations Yesterday, Today and Tomorrow. In Series on Nonlinear Science: Series A, World Scientific, Singapore, 2009.

[3] R. Kilic. A Practical Guide for Studying Chua's Circuits. In Series on Nonlinear Science: Series A, World Scientific, Singapore, 2010.

[4] R. Senani and S.S. Gupta. Implementation of Chua's chaotic circuit using current feedback op-amps. Electronics Letters, 47(1):829-830, 1998.

[5] L.A.B. Torres and L.A. Aguirre. Inductorless Chua's circuit. Electronics Letters, 36(23):1915-1916, 2000.

[6] Ö. Morgül. An RC realization of Chua's circuit family. IEEE Trans. Circuits Sys., 47(9):1424-1430, 2000.

[7] M.P. Kennedy. Robust op amp realization of Chua's circuit. Frequenz, 46(3-4):66-80, 1992.

[8] Chua's circuit diagrams, equations, simlulations and how to build. http://www. chuacircuits. com/. Accessed: 2015-03-07.

[9] Instructables - DIY how to make instructions. http: / /www. instructables. com/. Accessed: 2015-0307.

[10] A. Knörig, R. Wettach, and J. Cohen. Fritzing: A tool for advancing electronic prototyping for designers. In Proc. of the 3rd Int. Conf. on Tangible and Embedded Interaction, pages 351-358. ACM, 2009.

[11] Chaos circuit. http://www. instructables.com/id/Chaos-Circuit/. Accessed: 2015-03-07.

[12] Soundcard scope. http://www.zeitnitz.eu/scope_en. Accessed: 2015-03-07.

[13] P. Tebbe. A review of the current status and challenges of virtual experimentation. In Proc. of the Amer. Soc. Eng. Education Conf., 2006. 
[14] D.J. Moore and D.R. Voltmer. Curriculum for an engineering renaissance. IEEE Transaction on Education, 46 (4):452-455, 2003.

[15] G. Gandhi, et al. Anyone can build Chua's circuit: Hands-on-experience with chaos theory for high school students. Int. J. of Bifurcation and Chaos, 19(4):1113-1125, 2009.

[16] E. Bilotta, E. Bossio, and P. Pantano. Chaos at school: Chua's circuit for students in junior and senior high school. Int. J. of Bifurcation and Chaos, 20(1):1-28, 2010.

[17] A. Byagowi and W. Kinsner. Implementation of a Chua circuit to demonstrate bifurcations and strange attractors in a class. Proc. 2012 Canadian Engineering Education Association, 2012.

[18] S.H. Strogatz. Nonlinear Dynamics and Chaos: With Applications to Physics, Biology, Chemistry, and Engineering. Westview press, 2014.

[19] L.D. Feisel and A.J. Rosa. The role of the laboratory in undergraduate engineering education. Journal of Engineering Education, 94(1):121-130, 2005.

[20] L.M. Triona and D. Klahr. Point and click or grab and heft: Comparing the influence of physical and virtual instruction materials on elementary school students' ability to design experiments. Cognition and Instruction, 21(2):149-173, 2003.

[21] D. Klahr, L.M. Triona, and C. Williams. Hands on what? The relative effectiveness of physical versus virtual materials in an engineering design project by middle school children. Journal of Research in Science Teaching, 44(1):183-203, 2007.

[22] Z.C. Zacharia, E. Loizou, and M. Papaevripidou. Is physicality an important aspect of learning through science experimentation among kindergarten students? Early Childhood Research Quarterly, 27:447-457, 2012.

[23] J. Macias-Guarasa, et al. A project-based learning approach to design electronic systems curricula. IEEE Trans. Edu., 49(3):389-397, 2006.

[24] J. Ma and L Jeffrey. Hands-on, simulated, and remote laboratories: A comparative literature review. ACM Computing Surveys, 38(3), 1-24, 2006.

[25] R.M. Felder and L.K. Silverman. Learning and teaching styles in engineering education. Engineering Education, 78(7):674-681, 1988.

[26] N.D. Fleming and C. Mills. Not another inventory, rather a catalyst for reflection. To Improve the Academy, 11: $137-155,1992$. 